\title{
Expanding the Differential During the COVID-19 Pandemic: Fatal West Nile Virus Neuroinvasive Disease
}

\author{
Stephen J. Titus, MD, Robert N. Suter, DO, Anh T. Hoang, DO, and Paul A. Figel, DO
}

The ever-evolving pandemic of Coronavirus disease 2019 (COVID-19) has the potential to drown out other viruses continuing to infect communities. To highlight this, we present 2 cases of fatal West Nile virus neuroinvasive disease that occurred within 2 weeks of each other. Since the first positive case of West Nile virus in the United States, there have been 2 epidemics in the past 2 decades, most often occurring in regions of North Texas and Southern California, which have been areas of high-incidence for COVID-19. It is important for the health care provider to recognize diagnostic biases and maintain broad differentials for the patient presenting with fever and other symptoms associated with COVID-19. (J Am Board Fam Med 2021;34:661-662.)

Keywords: California, Communicable Diseases, CoVID-19, Family Medicine, Hospitalists, Pandemics, Texas, West Nile Virus

\section{Introduction}

Amid the COVID-19 pandemic, it is important to remember that many viruses, such as West Nile virus $(\mathrm{WNV})$, are still present. WNV infection is usually self-limited. However, less than $1 \%$ of cases develop neuroinvasion, which presents in 1 of 3 forms: meningitis, encephalitis, or acute flaccid paralysis. COVID-19 is similar to WNV infection in that they both have a high incidence in the southwestern United States, have a higher virulence pattern in older populations, and can present with the symptoms of fever and altered mental status. To illustrate this, we discuss 2 cases of fatal neuroinvasive $\mathrm{WNV}$ in elderly adults that presented within the same week to our service in Dallas, Texas.

This article was externally peer reviewed.

Submitted 8 December 2020; revised 10 February 2021; accepted 12 February 2021.

From the Family Medicine Residency, Baylor University Medical Center, Dallas, TX (SJT, RNS, ATH, PAF); Texas A\&M School of Medicine, College Station, Bryan, TX (SJT, RNS).

Funding: None.

Conflict of interest: None.

Corresponding author: Stephen J. Titus, MD, FAAFP, Baylor University Medical Center, GME 3500 Gaston Ave, 6th Fl Roberts, Dallas, TX 75246 (E-mail: stephen.titus@ bswhealth.org).

\section{Patient case 1}

An 88-year-old female with stage III chronic kidney disease (CKD), hypertension, and hyperlipidemia presented with altered mental status, diarrhea, nausea, vomiting, cough, and fever $\left(104^{\circ} \mathrm{F}\right)$. The initial workup included polymerase chain reaction (PCR) for the presence of SARS-CoV-2, blood cultures, and urine cultures, all of which were negative. She received empiric ceftriaxone, vancomycin, and acyclovir. Computed tomography (CT) of the head was unremarkable. A lumbar puncture revealed normal glucose, an elevated protein level $(77 \mathrm{mg} / \mathrm{dL}$; normal range, $15 \mathrm{mg} / \mathrm{dL}$ to $45 \mathrm{mg} / \mathrm{dL}$ ), and a lymphocytic pleocytosis with a white blood cell count of $68 / \mu \mathrm{L}$. Cerebral spinal fluid (CSF) cultures and a CSF meningitis panel (Table 1) were unremarkable. Despite treatment, her symptoms persisted. On day 7 of admission, she became bradycardic with a heart rate of 23 and expired. Postmortem, the serum WNV IgM drawn on the second day of admission returned positive, confirming WNV neuroinvasive disease.

\section{Patient case 2}

An 84-year-old female with a history of stroke presented with malaise, weakness most pronounced in the lower extremities, and confusion. Her family reported a recent history of an upper respiratory 
Table 1. Pathogens Tested in Meningitis Panel for Patient Case 2

Cytomegalovirus

Enterovirus

Herpes simplex virus type 1

Herpes simplex virus type 2

Human herpesvirus 6

Human parechovirus

Varicella zoster virus

Escherichia coli $\mathrm{K} 1$

Haemophilus influenza

Listeria monocytogenes

Neisseria meningitidis

Streptococcus agalactiae

Streptococcus pneumoniae

Cryptococcus neoformans/Cryptococcus gattii

infection but without known sick contacts, recent travel, fever, or insect bites. The initial workup including complete blood count, complete metabolic panel, thyroid-stimulating hormone, ammonia levels, and SARS-CoV-2 PCR was unremarkable. Empiric acyclovir and broad-spectrum antibiotics were initiated. CT and magnetic resonance imaging (MRI) of the head showed no acute intracranial pathology and nonspecific white matter changes. CSF analysis indicated lymphocytic pleocytosis with normal glucose, elevated protein of $108 \mathrm{mg} / \mathrm{dL}$, and no organisms. A meningitis panel was negative (Table 1). However, the CSF was positive for WNV IgG and IgM. Despite supportive care, the patient expired from $\mathrm{WNV}$ encephalitis.

\section{Discussion}

These cases contrast a typical and atypical presentation of $W N V$ with neuroinvasion. The first patient presented with typical symptoms of WNV infection, such as fever and gastrointestinal symptoms. ${ }^{1}$ The second patient presented with only malaise, weakness, and confusion. Both patients were geriatric, presented in the summer months with worsening altered mental status, and had CSF revealing lymphocytic pleocytosis.

Only $20 \%$ of those infected with $\mathrm{WNV}$ present with fever. ${ }^{1}$ Insect bites or recent travel in the patient's history should raise clinical suspicion. Initial neuroimaging may be negative as abnormal MRI findings may not be seen for several weeks, if at all. ${ }^{2}$ As a normal CSF cell count does not exclude WNV encephalitis, ${ }^{3}$ confirmation of diagnosis can be made by CSF PCR. ${ }^{4}$

The treatment for $\mathrm{WNV}$ is supportive care. Prognosis is related to comorbid conditions, such as diabetes, hypertension, and renal disease, all of which increase the probability of $\mathrm{WNV}$ neuroinvasion. ${ }^{5}$

\section{Conclusion}

While health care providers remain vigilant in recognizing COVID-19, we must continue to have an expansive differential that includes $\mathrm{WNV}$, which can reach epidemic levels in the summer and fall. It is important to educate patients on the use of mosquito repellent, longsleeve clothing, and the removal of standing water to help reduce risks posed by this preventable infectious disease.

To see this article online, please go to: http://jabfm.org/content/ 34/3/661.full.

\section{References}

1. Bai F, Thompson EA, Parminder JSV, Leis AA. Current understanding of West Nile virus clinical manifestations, immune responses, neuroinvasion, and immunotherapeutic implications. Pathogens 2019;8:193.

2. Sejvar JJ. Clinical manifestations and outcomes of West Nile virus infection. Viruses 2014;6:606-23.

3. Nash D, Mostashari F, Fine A, et al. 1999 West Nile Outbreak Response Working Group. The outbreak of West Nile virus infection in the New York City area in 1999. N Engl J Med 2001;344:1807-14.

4. Tyler KL, Pape J, Goody RJ, Corkill M, Kleinschmidt-DeMasters BK. CSF findings in 250 patients with serologically confirmed West Nile virus meningitis and encephalitis. Neurology 2006;66: 361-5.

5. Bode AV, Sejvar JJ, Pape WJ, Campbell GL, Marfin AA. West Nile virus disease: a descriptive study of 228 patients hospitalized in a 4-county region of Colorado in 2003. Clin Infect Dis 2006;42:1234-40. 